\title{
Gallstone ileus- An uncommon cause of bowel obstruction: A case report and review of literature.
}

\author{
Gopalakrishnan $\mathrm{G}^{1}$, Debasis Naik ${ }^{1}, \mathrm{~V}$. Bhandari ${ }^{2}, \mathrm{M}$ Tandon $^{3}$ \\ ${ }^{I}$ (Post-Graduate, Department of General Surgery, V.M.M.C and Safdarjung Hospital, New Delhi, India.) \\ ${ }^{2}$ (Associate Professor, Department of General Surgery, V.M.M.C and Safdarjung Hospital, New Delhi, India.) \\ ${ }^{3}$ (Senior Resident, Department of General Surgery, V.M.M.C and Safdarjung Hospital, New Delhi, India.)
}

\begin{abstract}
Gallstone ileus occurs when gallstone migrates from the gallbladder to the bowel through a cholecysto-enteric fistula, causing mechanical bowel obstruction. It is an uncommon cause of bowel obstruction. It occurs almost exclusively in the elderly, and accounts for $25 \%$ of mechanical small bowel obstruction in patients over the age of 65 years. Clinical diagnosis of gallstone ileus is difficult and usually depends on the radiographic findings. We present a case of 70 year old female with history of unstable angina, who presented with small bowel obstruction owing to a large gallstone in ileum.
\end{abstract}

Keywords: Gallstone ileus, Bowel obstruction, Cholecysto-enteric fistula, Enterolithotomy.

\section{Introduction}

Gallstone ileus, first described by Dr. Erasmus Bartholin in 1654, is a mechanical obstruction caused by the impaction of one or more gallstones within the lumen of any part of the gastrointestinal tract. It is a rare disease and accounts for about $1 \%$ to $3 \%$ of mechanical ileus of the small bowel, but for $25 \%$ of all small bowel obstructions in patients older than 65 years[1]. In patients with cholelithiasis only $0.3-0.5 \%$ develop gallstone ileus[2]. We report a case of 70 year old female with history of unstable angina, who presented with small bowel obstruction owing to large gallstone in ileum.

\section{Case Report}

A 70 years old woman presented to our emergency department with complaints of abdominal pain, distension, vomiting and constipation for 3days. She had similar complaints for 1 day, 5 days back. She had history of unstable angina 6 months back. She had no other comorbidities or history of surgery.

On physical examination, she was conscious, dehydrated, pulse rate was $120 / \mathrm{min}$, and blood pressure was $98 / 60 \mathrm{mmHg}$. Her abdomen was tense, distended, tenderness was present all over the abdomen, there was no guarding or rigidity. Bowel sounds were reduced. Digital rectal examination revealed empty rectum. Resuscitation was started immediately. X-ray Abdomen film showed dilated small bowel loops along with few air-fluid levels and there was no free gas under diaphragm. Ultrasonography abdomen revealed edematous and dilated small bowel loops with sluggish peristalsis along with mild ascites. Diagnosis of sub-acute intestinal obstruction was made and patient was put on conservative treatment.

Blood investigation showed, $\mathrm{Hb} \%-14.7 \mathrm{gm} / \mathrm{dl}$, WBC - 16,200/cubic millimetre of which $87 \%$ were polymorphs. Her Serum electrolytes, Renal function test and Liver function tests were normal. There was no improvement in her general condition with time and her obstruction was not relieved. NCCT abdomen revealed, proximal ileal intraluminal stone like density $(3 \mathrm{~cm}$ in diameter) at transit point with mural thickening and features of acute small intestinal obstruction with minimal pneumobilia and air density in gallbladder, suggestive of gallstone ileus(fig1\&2).

Emergency exploratory laparotomy was done. Intra-operatively, a gallstone of size $3 \times 2.5 \mathrm{~cm}$ was found obstructing the proximal ileal lumen, causing dilatation of proximal bowel loops and collapse of distal bowel loops(Transition point)(fig3\&4). Cholecysto-enteric fistula couldn't be identified due to dense peri-cholecystic adhesion. Proximal longitudinal enterolithotomy with decompression of the proximal bowel was done. But the patient expired on first post-operative day due to myocardial infarction.

\section{Discussion}

Gallstone ileus is an uncommon condition that may result when a gallbladder stone enters into the intestinal tract, usually as a result of an internal fistula between the gallbladder and duodenum[3].

The formation of a fistula between the gallbladder and the duodenum may allow a gallstone to enter the intestinal tract. Cholecystoduodenal fistula is the most frequent (75\%), followed by chylecystocolic fistula(10$20 \%$ ), and a variety of other types (15\%). Spontaneous entero-biliary fistula occurs secondary to biliary disease, and diseases of adjacent structures. These are usually associated with gallstone but have also been reported with 
Gallstone ileus- An uncommon cause of bowel obstruction: A case report and review of literature.

peptic ulcer disease, abdominal trauma, Crohn's disease, and malignancy of biliary tract, bowel, and head of pancreas[4].

The terminal ileum is the most frequent site of obstruction [3]. However, it may be found in duodenum causing Bouveret's syndrome[2]. Other points of obstruction, being jejunum (30\%) and colon (2.5\%).

The classical radiologic triad or Rigler triad of pneumobilia, small bowel obstruction and ectopic gallstone is specific for this disease, but only $9-14 \%$ of patients have a clear-cut Rigler's triad[5].

Ultrasonography has been shown to be more helpful than plain films in diagnosing gallstone ileus. Ripolles $\mathrm{T}$ et al found that abdominal sonography demonstrated bowel dilatation (44.44\%), extraluminal fluid (14.81\%), ectopic stones (14.81\%), gallbladder abnormalities (37.04\%), and pneumobilia (55.56\%)[6].

Computed tomography (CT) scans may also provide useful information if the diagnosis is in doubt. CT is useful for estimating the size and number of impacted gallstones and the transition point between dilated and collapsed bowel[7].

Gallstone ileus usually requires emergency surgery to relieve intestinal obstruction. There is no uniform surgical procedure for this disease because of its low incidence[8]. Enterolithotomy alone is the preferred surgery more than enterolithotomy combined with cholecystectomy[9]. Pavlidis TE et al compared the mortality from one-stage procedure (entrolithotomy plus fistula repair and cholecystectomy) (16.9\%) versus enterolithotomy alone $(11.7 \%)[10]$.

Recently, laparoscopy guided enterolithotomy has become the preferred surgical approach in treating gallstone ileus[11]. Additionally, the non-surgical treatment of gallstone ileus has been suggested, including endoscopic removal and shock wave lithotripsy, but this depends on the location of obstruction[12,13].

The prognosis of gallstone ileus is usually poor and worsens with age. This is largely due to delayed diagnosis and concomitant conditions such as cardio-respiratory disease, obesity and diabetes mellitus[1].

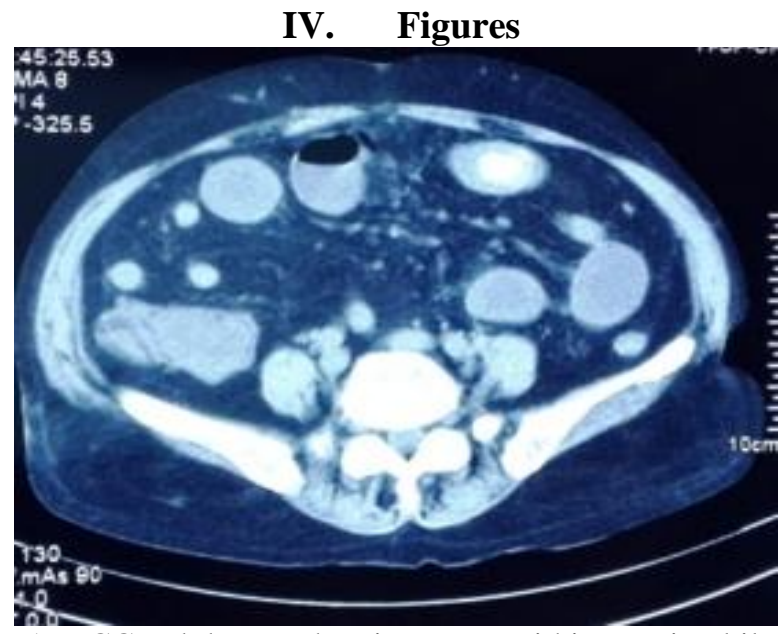

Fig 1: NCCT abdomen showing stone within proximal ileum

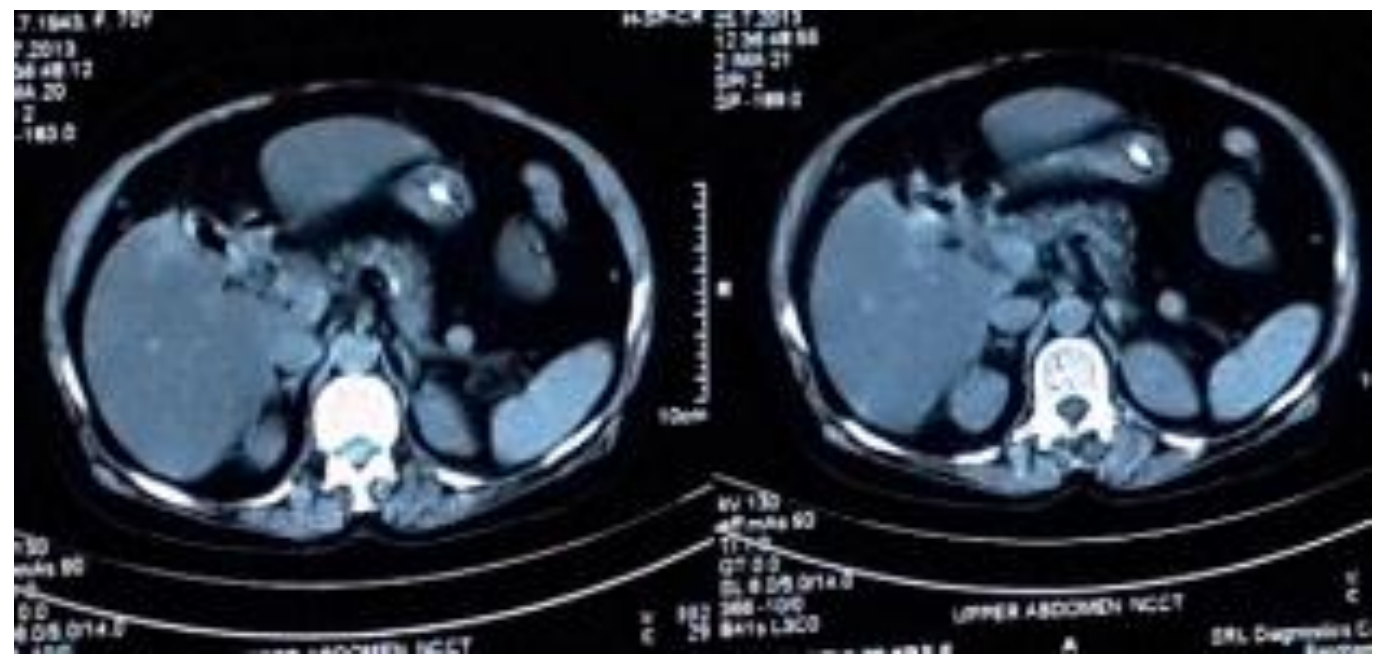

Fig 2: NCCT abdomen showing gallstone ileus and pneumobilia. 


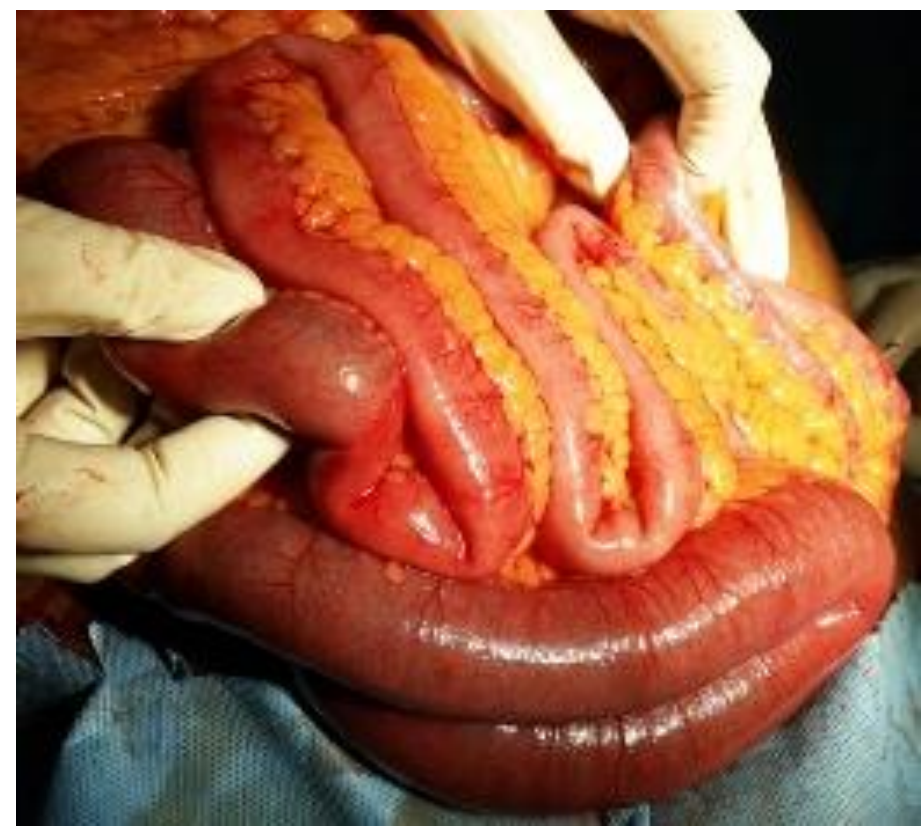

Fig 3: Transition point due to impacted gallstone

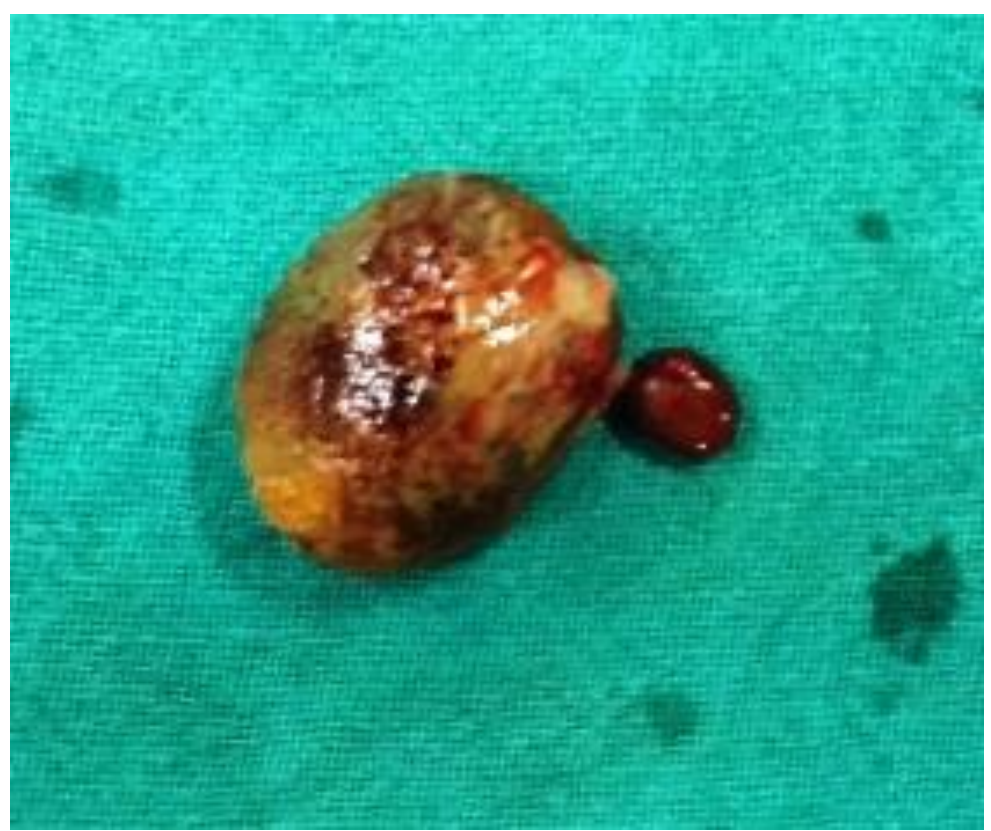

Fig 4: Extracted gallstone $(3 \times 2.5 \mathrm{~cm})$ through longitudinal enterolithotomy.

\section{Conclusion}

In conclusion, delay in achieving diagnosis of gallstone ileus is not uncommon. If left undetected it leads to increased mortality. A high index suspicion in older patients with intermittent gastro-intestinal symptoms should trigger appropriate sensitive investigation like computed tomography of the abdomen for early diagnosis and management in order to reduce mortality.

\section{References}

[1]. Reisner RM, Cohen JR. Gallstone ileus: A review of 1001 reported cases. Am Surg. 1994; 60: $441-446$.

[2]. Chen-Wang C, Shou-Chuan S, Shee-Chau L, Cheng-Hsin C.Gallstone ileus: A disease easily ignored in the elderly. Int J Gast enterology. 2008; 2(1): 18-21.

[3]. Chatterjee S, Tamonas C, Goutan G, Ambar G.Gallstone ileus an atypical presentation and unusual location.Int J Surj. 2008; 6(6):e55-e56.

[4]. Hernandez C, Heuman D, Vlahcevid ZR. Pathophysiology of disease associated with deficiency of bile acids. Principles and practice of Gastroentrology and hepatology. New York: Elsevier Science. 1988; 348-95.

[5]. Elabsi M, Amraoui M, Errougani A, Chkof MR. Diagnosis and treatment: gallstone ileus.Digestive and liver disease. 2007; 39:180181. 
Gallstone ileus- An uncommon cause of bowel obstruction: A case report and review of literature.

[6]. Ripolles T,Miguel-Dasit A, Errando J, Morote V, Gomez-Abril SA, Richart J. Gallstone ileus: Increased diagnostic sensitivity by combining plain film and ultrasound.Abdom Imaging. 2001; 26: 401-5.

[7]. Lassandro F, Romano S, Ragozzino A, Rossi G, Valente T, Ferrara I, Romano L, Grassi R. Role of helical CT in diagnosis of gallstone ileus and related conditions. AJR Am J Roentgenol. 2005; 185: 1159-1165.

[8]. Syme RG. Management of gallstone ileus. Can J Surg. 1989; 32: 61-4.

[9]. Tan YM,Wong WK, Ooi LL. A comparison of two surgical strategies for the emergency treatment of gallstone ileus. Singapore Med J. 2004; 45: 69-72

[10]. Pavlidis TE, Atmatzidis KS, Papaziogas TB. Management of gallstone ileus. J Hepatobiliary Pancreat Surg. 2003; 10: 299-302.

[11]. Frankalin ME Jr, Doman JP, Schuessler WW. Laparoscopic treatment of gallstone ileus:a case report and review of literature. J Laparoendosc Surg. 1994; 4: 265-272.

[12]. Dumonceau JM, Delhaye M, Deviere J, Baize M, Cremer M.Endoscopic treatment of gastric outlet obstruction caused by a gallstone(Bouverets syndrome) after extracorporeal shock wave lithotripsy.Endoscopy. 1997; 29: 319-21

[13]. Meyenberger C, Michel C, Metzger U, Koelz HR.Gallstone ileus treated by shock wave lithotripsy.Gastrointest Endosc. 1996; 43: 508-11. 